\title{
Prevalence of Microorganisms and Antibiotic Sensitivity Among Patients with Diabetic Foot Ulcer in Sulaimani City, Iraq
}

\author{
Aso Nasih Qadir ${ }^{*}$, Bakhtiar Mohamed Mahmoud ${ }^{2}$, Taha Othman Mahwi ${ }^{3}$, Delman Mohammed Raoof \\ Arif Al-Attar ${ }^{3}$, Safeen Othman Mahmood ${ }^{3}$
}

${ }^{1}$ Ministry of Health, Kurdistan Regional Government, Sulaymaniyah, Iraq

${ }^{2}$ Ministry of Higher Education and Scientific Research, Training Center of Sulaymaniyah, Sulaymaniyah, Iraq

${ }^{3}$ College of Medicine, University of Sulaymaniyah, Sulaymaniyah, Iraq

*Corresponding Author: Aso Nasih Qadir, M.D., Internal Medicine Board Candidate, Ministry of Health, Kurdistan Regional Government, Sulaymaniyah, Iraq. Tel: +964-7705071364, Email: aso.nasih@gmail.com

Received June 21, 2019; Accepted March 13, 2020; Online Published June 19, 2020

\begin{abstract}
Background: Diabetic foot ulcers (DFUs) are responsible for days of costly hospitalization and are the major cause of medical lower limb amputations. Determining the appropriate antimicrobial therapy for DFUs is highly dependent on recognizing the microorganisms that cause them. Many reports have indicated that there has been a remarkable increase in antibiotic resistance. Objectives: The present study examined various cultures of patients with DFUs to detect the prevalence of microorganisms and their antibiotic sensitivity profiles.

Methods: This cross-sectional study was carried out on 92 patients with DFUs admitted to Shar Teaching Hospital and Shahid Hemin Teaching Hospital. Wagner Classification was used to assess the severity and location of the DFUs. Patients were asked questions about their practices of foot care and hygiene, and their answers were recorded. Samples required for testing were taken using sterile swabs.

Results: A total of 100 microorganisms were isolated from 92 patients with DFUs, 10 of which were polymicrobial and 2 were culture-negative. There was a highly significant association between the isolated gram-negative microorganisms and higher grades of DFU $(P<0.001)$. A highly significant association was also observed between bad patient knowledge of hygiene practices and gram-negative microorganisms $(P<0.001)$. Osteomyelitis was present in $40(43.4 \%)$ patients.

Conclusion: Among gram-positive and gram-negative bacteria, Staphylococcus spp. and E. coli were, respectively, the most frequent organisms isolated. The antibiotic imipenem was found to be effective against microorganisms. Tetracycline, erythromycin, and ceftriaxone, however, were highly resistant to antibiotics. To sum up, since different microorganisms are involved and multidrugresistant strains might emerge, clinicians are recommended to take cultures into account before they initiate empirical therapy. Keywords: Diabetes, Foot Ulcer, Gram-positive Bacteria, Antibiotic Therapy
\end{abstract}

\section{Background}

As a serious complication associated with diabetes, diabetic foot can seriously affect the quality of life in patients. ${ }^{1}$ According to a report of the World Health Organization (WHO), the incidence of diabetes mellitus (DM) is on a steady rise, particularly in developing countries. Thus, determining what microorganisms are involved in such infections is essential to determining an appropriate antimicrobial therapy. ${ }^{2}$

The burden of diabetic foot disease is expected to rise given the increasing global prevalence of type 2 diabetes mellitus (T2DM). ${ }^{3}$ Diabetic foot ulcer (DFU) causes significant morbidity and mortality in those patients who develop this complication. ${ }^{4}$ About 382 million people worldwide suffer from diabetes, and it has been estimated that this number will increase to 592 million by the year $2035 .{ }^{4}$ It has also been stated that $15 \%$ of patients with DM will develop DFU at some point in their lives. ${ }^{3,5}$ Clearly, patients with uncontrolled diabetes are at high risk for developing complications like pedal ulcer, diabetic retinopathy, diabetic nephropathy, and diabetic neuropathy. Moreover, if DFUs are not treated promptly, they may frequently lead to amputations., ${ }^{3,6}$ DFUs are responsible for more days of costly hospitalization than any other diabetes-related complication and are the major cause of medical lower limb amputations. ${ }^{7}$ DFUs are commonly classified according to Wagner's classification system, which assesses ulcer depth and the presence of osteomyelitis or gangrene (Table 1).

Depth of the ulcer is the most important risk predictor

Copyright (C) 2020 The Author(s). This is an open-access article distributed under the terms of the Creative Commons Attribution License (http:// creativecommons.org/licenses/by/4.0), which permits unrestricted use, distribution, and reproduction in any medium, provided the original work is properly cited. 
Table 1. Standard Treatment of Diabetic Foot According to Wagner's Classification ${ }^{8}$

\begin{tabular}{lll}
\hline Grade & - & - \\
\hline 0 & Foot at risk & Prevention \\
1 & Localized, superficial ulcer & Antibiotics and glycemic control \\
2 & Deep ulcer to bone, ligament, or joint & Debridement, antibiotics, and glycemic control \\
3 & Deep abscess, osteomyelitis & Debridement, some form of amputation \\
4 & Gangrene of toes, forefoot & Wide debridement and amputation \\
5 & Gangrene of entire foot & Below knee amputation \\
\hline
\end{tabular}

of amputation and is usually caused by neuropathy, peripheral arterial disease, decreased immunity, and pressure overload. 5 Other factors associated with amputation include age, male gender, and long duration of diabetes. ${ }^{10,11}$ The wound surface can be colonized by a wide range of microorganisms, and it may be polymicrobial. ${ }^{3}$ In DFUs, correct antibiotic usage based on cultures and sensitivity is needed for proper management. ${ }^{5}$ Good management of the infection can reduce the incidence of infection-related morbidities, the need for and duration of hospitalization, and the incidence of major limb amputation. Early identification of the lesions, prompt initiation of appropriate antibiotic therapy, aggressive surgical debridement of necrotic soft tissue and bone, and modification of host factors are all equally important for a successful clinical outcome. ${ }^{12-14}$

Patient knowledge and practice of foot care are crucial to preventing foot ulcer formation. ${ }^{15,16}$ However, several studies found that most patients do not receive adequate information regarding foot care practices. ${ }^{4}$ Today, there are specialized diabetic foot units where proper management of DFU (like empiric antibiotic therapy) is carried out after antibiotic sensitivity testing and while the results of culture and sensitivity testing is pending. ${ }^{15}$ The most common isolates from DFUs are gram-positive cocci, especially Staphylococcus aureus. The advised empirical treatment is a narrow spectrum for low-grade ulcers and a broader spectrum for higher grades. ${ }^{16}$

\section{Objectives}

The present study aimed to examine the prevalence of microorganisms in patients with DFUs and evaluate their drug sensitivity patterns.

\section{Methods}

3.1. Patient Selection; Inclusion and Exclusion Criteria

This cross-sectional study was carried out on 92 cases with DFUs admitted to medical and orthopedic wards of Shar Teaching Hospital and Shahid Hemin Hospital in Sulaimani, the Kurdistan region of Iraq, from September 2018 to April 2019. Patients who were diagnosed with type 2 or type $1 \mathrm{DM}$ (HbA1c more than $6.5 \%$ based on American Diabetic Association criteria, ${ }^{17}$ treated with oral anti-diabetic medication or insulin, and had DFUs were included in the study. Cases who were treated with antibiotics before hospital admission, those who had impaired glucose tolerance tests, those with traumatic foot ulcers, patients who refused to provide informed consent, and those who were under 18 years of age were excluded from the study. Ethical consent was obtained from all participating cases. Demographic data (including gender, weight, and height), type of diabetes, type of treatment, duration of diabetes, and comorbidities was collected.

\subsection{Sampling and Antibiotic Sensitivity Testing 3.2.1. Sampling Collection}

First, sampling collection was carried out based on the grade of ulcer:

- Grade 1: Subcutaneous aspirate after proper skin disinfectant use;

- $\quad$ Grade 2: Skin swabbing and subcutaneous aspirate;

- Grades 3 and 4: Wound swab, pus collection, scraping, and tissue sampling for gram stain, fungal, and cultural sensitivity.

Sterile cotton swabs, syringes, and the sterile containers were used to take samples following disinfecting (for grade 1) of the skin surrounding the ulcer. Pus samples were taken from the deepest parts of the base of the ulcer by rubbing it with a sterile swab in a circular motion. All procedures were performed aseptically. Sampling was not done during the holidays.

\subsubsection{Transportation}

Samples were immediately transported to the microbiology laboratory for processing without storage.

\subsubsection{Processing}

Before smear staining, the samples were cultured to avoid contamination culture. This procedure included:

1. Aerobic culture at $37^{\circ} \mathrm{C}$ on blood and McConkey agar, $\mathrm{CO} 2$ enhanced culture on chocolate agar for fastidious bacteria and Sabouraud Dextrose agar as special fungal isolation media (at both $37^{\circ} \mathrm{C}$ and $25^{\circ} \mathrm{C}$ );

2. Anaerobic enhanced culture in thioglycolate-enriched broth (supportive for anaerobic and microaerophilic agents);

3. Part of the swab, pus, or tissue sample was put inside a container with thioglycolate to support anaerobic growth.

All samples were incubated for 48 hours on Petri dishes in each atmosphere. Thioglycolate broth was subcultured after 48 hours of incubation for another 48 hours' duration 
on similar agars.

Each positive sample was processed based on the following logic:

1. Gram stain on a colony to determine whether it is gram-positive or gram-negative.

2. Using VITEK II identification and antibiotic sensitivity testing(AST).

3. AST panel items were used based on the availability of (automated VITEK II AST card) with different codes that may include different items that are not uniform.

\subsection{Degree of Adherence to Medications}

The degree of adherence to medications was determined according to the Morisky Green Levine Medication Adherence Scale (Table 2). ${ }^{18}$

Scores gained from the MGLS range from 0-4, and each of the four items is in a (yes/no) format. One point is scored for each positive response; 1 point is given for a "yes" answer, and 0 point for a "no" answer. A score of 0 indicates high adherence; a score of 1 or 2 illustrates intermediate adherence, and a score of 3 or 4 indicates low adherence.

\subsection{Patients' Knowledge about Foot Care and Hygiene} Practices

This study employed a diabetic foot care questionnaire designed by Hasnain and Sheikh (2009) which comprised a set of 15 yes-or-no questions about knowledge and practices (Table 3). Each 'yes' answer carried one (1) point and each 'no' answer carried zero points. The level of knowledge and practice, whether good or poor, was determined based on the median score of each category. Scores higher than the median were considered good, and scores lower than the median were considered bad.

\subsection{Laboratory Investigations}

Laboratory investigations were performed during admission after blood samples were taken aseptically from upper limb peripheral veins and included whole blood count, $\mathrm{HbA1c}$, and lipid profile (triglycerides, cholesterol, low-density lipoprotein) after 12 hours of fasting.

Based on the criteria of the American Diabetes Association, ${ }^{17}$ an $\mathrm{HbA} 1 \mathrm{c}$ value less than $7 \%$ was regarded as controlled and more than $7 \%$ as uncontrolled. According to the WHO, ${ }^{20}$ hemoglobin levels of less than $12.9 \mathrm{~g} /$ $\mathrm{dL}$ for males and $11.9 \mathrm{~g} / \mathrm{dL}$ for females were regarded as anemia. Based on criteria from the American College of Endocrinology, ${ }^{21}$ triglycerides of less than $150 \mathrm{mg} / \mathrm{dL}$, total cholesterol of less than $200 \mathrm{mg} / \mathrm{dL}$, and low-density lipoprotein (LDL) of less than $130 \mathrm{mg} / \mathrm{dL}$ were considered normal.

\subsection{Statistical analysis}

The collected data was entered in Microsoft Excel and

Table 2. Patients Self-reported Adherence to diabetic medications according to the Morisky Green Levine Medication Adherence Scale (MLGS) ${ }^{18}$

\begin{tabular}{|c|c|c|c|}
\hline & Question & Yes & No \\
\hline Q 1 & Do you ever forget to take your diabetic medication?* & & \\
\hline Q 2 & Are you careless at times about taking your diabetic medication?* & & \\
\hline Q 3 & When you feel better, do you sometimes stop talking your diabetic medication?* & & \\
\hline Q 4 & Sometimes if you feel worse when you take your diabetic medication, do you stop taking it?* & & \\
\hline \multicolumn{4}{|c|}{ Note: * Negatively worded question } \\
\hline
\end{tabular}

Table 3. Diabetic Foot Care Questionnaire Assessing Knowledge and Practices ${ }^{19}$

\begin{tabular}{|c|c|c|}
\hline & Foot care measures & Yes/No \\
\hline 1 & Importance of taking antidiabetic treatment to prevent complications & \\
\hline 2 & Daily washing of the feet & \\
\hline 3 & Using warm water for washing/bathing & \\
\hline 4 & Checking the temperature of the water before using & \\
\hline 5 & Drying the feet after washing & \\
\hline 6 & Talcum powder usage for keeping interdigital spaces dry & \\
\hline 7 & Keeping the skin of the feet soft to prevent dryness & \\
\hline 8 & Lotion not to be applied in the interdigital spaces & \\
\hline 9 & Daily change of socks & \\
\hline 10 & Trimming nails of feet straight with care & \\
\hline 11 & Inspection of feet once a day by respondents & \\
\hline 12 & Wearing comfortable shoes & \\
\hline 13 & Checking the shoes from inside before putting them on & \\
\hline 14 & Not walking barefoot & \\
\hline 15 & Warning signs for which consultation is required & \\
\hline
\end{tabular}


later coded for statistical analysis using Statistical Package for the Social Sciences (SPSS, version 22). Descriptive approaches were used to calculate frequencies, percentages, and means. A $P$ value of $\leq 0.05$ was regarded as statistically significant, and a $P$ value of $<0.001$ was considered highly significant statistically.

\section{Results}

This study included 92 diabetic foot patients with a mean age of $59.5 \pm 12.1$ years. There were more male patients than females $(56.5 \%$ vs. $43.5 \%)$. The majority of the patients (95.6\%) had T2DM. The mean DM duration was 14.6 \pm 7.9 years. The mean body mass index of diabetic foot patients was $25.6 \pm 4.1 \mathrm{~kg} / \mathrm{m}^{2}$. The main treatment was drugs (76.08\%), followed by insulin (17.3\%) and combined drugs and insulin (6.5\%). Patient adherence to treatment was commonly low (64.1\%), and smoking history was positive in $26.08 \%$ of cases. Past medical history was positive in $61.9 \%$ of the patients, and history of an ulcer was positive in $52.2 \%$ of them (Table 4 ).

Most DFUs were in grades $2(39.1 \%)$ and 3 (28.2\%), respectively. Ulcer site in most cases $(73.9 \%)$ was the right foot. The knowledge of foot care was bad in $63.04 \%$ of diabetic foot patients (Table 5).

X-ray findings of $56.5 \%$ diabetic foot patients were normal, while $43.4 \%$ of cases had osteomyelitis (26 cases were grade 3 , while 14 cases were grade 4 ). The mean white blood cell count (WBC) in diabetic foot patients was $12.1 \times 10^{3}$, hemoglobin $11 \mathrm{~g} / \mathrm{dL}$, total cholesterol 183 $\mathrm{mg} / \mathrm{dL}$, triglyceride $197.4 \mathrm{mg} / \mathrm{dL}$, and LDL (92.2 mg/dL) (Table 6).

A total of 100 positive microbial agents were isolated from swabs taken from the diabetic foot patients. Two diabetic foot patients had no growth, while 10 had polymicrobial infections. The gram-positive microorganisms represented $53 \%$ of the microorganisms isolated, with the first three most frequent of them being S. aureus (54.7\%), Staphylococcus epidermidis (22.6\%), and Enterococcus faecalis (15.1\%). Gram-negative microorganisms represented $47 \%$ of the total isolated microorganisms, with the first three most frequent of them being E. coli (32\%), Klebsiella (17\%), and Proteus mirabilis (17\%) (Table 7).

Antibiotic sensitivity in descending order was higher for the following antibiotics: imipenem (100\%), moxifloxacin (100\%), teicoplanin (100\%), daptomycin (100\%), vancomycin (95.7\%), and meropenem (90.2\%), while the resistance was high for the following antibiotics: erythromycin $(79.5 \%)$, tetracycline $(78.1 \%)$, ampicillin (72.7\%), and ceftriaxone (72.1\%) (Table 8).

Regarding the severity of the diabetic foot lesions, there was a highly significant association between the isolated gram-negative microorganisms and higher grades of DFUs $(P<0.001)$. Regarding the site of foot ulcers, no significant differences were observed between diabetic foot patients with gram-positive microorganisms and diabetic foot patients with gram-negative microorganisms $(P=0.2)$. A highly significant association was observed between
Table 4. General Characteristics Of Diabetic Foot Patients

\begin{tabular}{|c|c|}
\hline Variable & No. $(\%)$ \\
\hline \multicolumn{2}{|c|}{ Age, mean \pm SD $(59.5 \pm 12.1$ years $)$} \\
\hline$<40$ & $6(6.5)$ \\
\hline $40-49$ & $11(11.9)$ \\
\hline $50-59$ & $32(34.7)$ \\
\hline $60-69$ & $23(25)$ \\
\hline$\geq 70$ & $20(21.8)$ \\
\hline \multicolumn{2}{|l|}{ Gender } \\
\hline Male & $52(56.5)$ \\
\hline Female & $40(43.5)$ \\
\hline \multicolumn{2}{|l|}{ Type of DM } \\
\hline Type 1 & $4(4.3)$ \\
\hline Type 2 & $88(95.6)$ \\
\hline \multicolumn{2}{|c|}{ DM duration, mean \pm SD (14.6 \pm 7.9 years $)$} \\
\hline$\leq 10$ years & $36(42.3)$ \\
\hline $11-20$ years & $45(48.9)$ \\
\hline$>20$ years & $11(11.9)$ \\
\hline \multicolumn{2}{|c|}{$\mathrm{BMI}$ mean $\pm \mathrm{SD}\left(25.6 \pm 4.1 \mathrm{~kg} / \mathrm{m}^{2}\right)$} \\
\hline Normal & $53(57.6)$ \\
\hline Overweight & $25(27.1)$ \\
\hline Obese & $14(15.2)$ \\
\hline \multicolumn{2}{|l|}{ Type of treatment } \\
\hline Drugs & $70(76.08)$ \\
\hline Insulin & $16(17.3)$ \\
\hline Drugs and insulin & $6(6.5)$ \\
\hline \multicolumn{2}{|l|}{ Adherence to medications } \\
\hline High adherence & $27(29.3)$ \\
\hline Intermediate adherence & $30(32.6)$ \\
\hline Low adherence & $35(38.1)$ \\
\hline \multicolumn{2}{|l|}{ Smoking } \\
\hline No & $56(60.8)$ \\
\hline Yes & $24(26.08)$ \\
\hline Ex & $12(13.03)$ \\
\hline \multicolumn{2}{|l|}{ Past medical history } \\
\hline Negative & $35(38.04)$ \\
\hline Positive & 57 (61.9) \\
\hline \multicolumn{2}{|l|}{ Previous history of ulcer } \\
\hline No & $44(47.8)$ \\
\hline Yes & $48(52.2)$ \\
\hline Total & $92(100.0)$ \\
\hline
\end{tabular}

Table 5. Characteristics of Diabetic Foot Ulcer

\begin{tabular}{lc}
\hline Variable & No. $\mathbf{( \% )}$ \\
\hline Grade of ulcer & $16(17.3)$ \\
Grade 1 & $36(39.1)$ \\
Grade 2 & $26(28.2)$ \\
Grade 3 & $14(15.2)$ \\
Grade 4 & \\
Site of ulcer & $68(73.9)$ \\
Right foot & $24(26.08)$ \\
Left foot & \\
Patient knowledge of foot care and hygiene practices & $58(63.04)$ \\
Bad & $34(36.9)$ \\
Good & $92(100.0)$ \\
\hline Total &
\end{tabular}


Table 6. Findings Regarding Diabetic Foot Patients $(n=92)$

\begin{tabular}{ll}
\hline Variable & No. $(\%)$ \\
\hline X-ray finding & \\
$\quad$ Normal & $52(56.5)$ \\
$\quad$ Osteomyelitis & $40(43.4)$ \\
White cell count, mean \pm SD $\left(12.1 \pm 0.5 \times 10^{3}\right)$ & \\
Hemoglobin, mean \pm SD $(11 \pm 2 \mathrm{~g} / \mathrm{dL})$ & $47(51.1)$ \\
$\quad$ No anemia & $45(48.9)$ \\
$\quad$ Anemia & \\
HbA1c, mean \pm SD $(9.9 \pm 1.9 \%)$ & $4(4.3)$ \\
$\quad$ Good control & $88(95.7)$ \\
$\quad$ Poor control & \\
Total cholesterol, mean $\pm S D(183 \pm 57.8 \mathrm{mg} / \mathrm{dL})$ & \\
Triglyceride, mean $\pm S D(197.4 \pm 82.5 \mathrm{mg} / \mathrm{dL})$ & \\
Low-density lipoprotein, mean $\pm \mathrm{SD}(92.2 \pm 35.4 \mathrm{mg} / \mathrm{dL})$ & \\
\hline
\end{tabular}

Table 7. Culture Findings of Gram +ve and - ve Microorganisms of Diabetic Foot Ulcers*

\begin{tabular}{lc}
\hline Variable & Gram +ve No. (\%) \\
\hline Staphylococcus aureus & $29(54.7)$ \\
Staphylococcus epidermidis & $12(22.6)$ \\
\hline Enterococcus faecalis & $8(15.1)$ \\
Staphylococcus chromogenes & $2(3.8)$ \\
Staphylococcus lentus & $2(3.8)$ \\
\hline Total & $53(53.0)$ \\
\hline & Gram -ve No. (\%) \\
\hline Morganella morganii & $4(8.5)$ \\
\hline Proteus mirabilis & $8(17.0)$ \\
Klebsiella & $8(17.0)$ \\
Escherichia coli & $15(32.0)$ \\
Providencia stuartii & $2(4.3)$ \\
Pseudomonas aeruginosa & $4(8.5)$ \\
Acinetobacter baumannii & $4(8.5)$ \\
Raoultella & $2(4.3)$ \\
Total & $47(47.0)$ \\
\hline Analzed by chisquar test. &
\end{tabular}

*Analyzed by chi-square test.

patients' bad knowledge and hygiene practices and gramnegative microorganisms $(P<0.001)$ (Table 9$)$.

\section{Discussion}

As the most severe complication of diabetes, foot ulceration affects diabetic patients who are not restricted to certain superficial underlying subcutaneous tissue. If diabetes is not controlled or if there is poor health self-care, diabetic foot ulceration may result. ${ }^{22,23}$ It is essential to identify the microorganisms responsible for DFU in order to determine the treatment for DFUs so as to prevent amputation. ${ }^{24}$

In the present study, more than half of the patients were men, and slightly over $6 \%$ of them were younger than 40 years of age. The fact that over $93 \%$ of patients were older than 40 years may be due to the fact that today, various factors, such as professional activities and lifestyle, that would cause the feet to tolerate more pressure have changed, and consequences are more commonly seen in middle-aged and older people. ${ }^{3}$ The present study showed
Table 8. Culture Sensitivity and Resistance Results of Antibiotics

\begin{tabular}{|c|c|c|}
\hline Antibiotic & Sensitive No. (\%) & Resistant No. (\%) \\
\hline Imipenem & $41(100.0)$ & $0(0)$ \\
\hline Moxifloxacin & $20(100.0)$ & $0(0)$ \\
\hline Teicoplanin & $17(100.0)$ & $0(0)$ \\
\hline Vancomycin & $45(95.7)$ & $2(4.3)$ \\
\hline Meropenem & $37(90.2)$ & $4(9.8)$ \\
\hline Piperacillin tazobactam & $24(88.9)$ & $3(11.1)$ \\
\hline Amikacin & $31(75.6)$ & $10(24.4)$ \\
\hline Levofloxacin & $30(56.6)$ & $23(43.4)$ \\
\hline Rifampicin & $16(53.3)$ & $14(46.7)$ \\
\hline Ceftazidime & $18(52.9)$ & $16(47.1)$ \\
\hline Gentamycin & $32(50.0)$ & $32(50.0)$ \\
\hline Ciprofloxacin & $28(50.0)$ & $28(50.0)$ \\
\hline Aztreonam & $8(50.0)$ & $8(50.0)$ \\
\hline Bactrim & $20(34.5)$ & $38(65.5)$ \\
\hline Amoxiclav & $12(34.3)$ & $25(65.7)$ \\
\hline Clindamycin & $12(32.4)$ & $25(67.6)$ \\
\hline Cefuroxime & $6(30.0)$ & $14(70.0)$ \\
\hline Cefepime & $6(28.6)$ & $15(71.4)$ \\
\hline Ceftriaxone & $12(27.9)$ & $31(72.1)$ \\
\hline Ampicillin & $12(27.3)$ & $32(72.7)$ \\
\hline Tobramycin & $3(25.0)$ & $9(75.0)$ \\
\hline Tetracycline & $7(21.9)$ & $25(78.1)$ \\
\hline Erythromycin & 8 (20.5) & $31(79.5)$ \\
\hline
\end{tabular}

Table 9. Distribution of Diabetic Foot Ulcer Characteristics According to Gram Stain Results

\begin{tabular}{|c|c|c|c|}
\hline Variable & Gram +ve No. $(\%)$ & Gram -ve No. (\%) & $P$ Value \\
\hline \multicolumn{4}{|c|}{ Grade of ulcer } \\
\hline Grade 1 & $8(8)$ & $8(8)(17.0)$ & \multirow{4}{*}{$<0.001^{*}$} \\
\hline Grade 2 & $26(26)(49.1)$ & $15(15)(31.9)$ & \\
\hline Grade 3 & 19 (19) (35.8) & $10(10)(21.3)$ & \\
\hline Grade 4 & $0(0)$ & $14(14)(29.8)$ & \\
\hline \multicolumn{4}{|l|}{ Site } \\
\hline Right & $41(77.4)$ & $31(66.0)$ & \multirow[t]{2}{*}{0.205} \\
\hline Left & $12(22.6)$ & $16(34.0)$ & \\
\hline \multicolumn{4}{|c|}{ Patient knowledge about foot care and hygiene practices } \\
\hline Bad & $23(43.4)$ & $41(87.2)$ & \multirow[t]{2}{*}{$<0.001^{*}$} \\
\hline Good & $30(56.6)$ & $6(12.8)$ & \\
\hline
\end{tabular}

*Significant

that most of the patients were of an elderly age and the male gender. Consistently, Sari et al documented that being elderly and being male were associated with DFUs. ${ }^{25}$ The mean duration of diabetes in the present study was 14.6 years, which is close to the mean duration of diabetes of 13.5 years reported in Iranian diabetic patients by Farshad et al. ${ }^{26}$ Mohammed et al stated that older age, long diabetes duration, multiple anti-diabetics, hard physical activity, and bad foot care are the common risk factors for the development of DFUs in Iraq. ${ }^{27}$

In the present study, DFU cultures revealed that $53 \%$ and $47 \%$ of the microorganisms were gram-positive and gramnegative, respectively. This finding is inconsistent with the 
results of Xie et al, whose study in China reported a higher prevalence of gram-negative bacteria in comparison to gram-positive bacteria isolated from DFUs (54.1\% vs. 45.9\%). ${ }^{28}$ However, the findings of the present study are similar to the results of Al-Hemedawi and colleagues' study in Iraq which found that gram-positive bacteria, particularly S. Aureus, were most common. ${ }^{29}$ Differences in microorganism cultures between different studies might be due to the variance in risk factors in developing foot ulcers among diabetic patients. The current study showed that $S$. aureus was the most prevalent gram-positive bacteria and E. coli was the prevalent gram-negative bacteria responsible for DFUs. These findings are consistent with the results of studies carried out by Al-Hemedawi in Iraq and Sekhar et al in India..$^{29,30}$

In the current study, microorganisms showed higher sensitivity to imipenem, moxifloxacin, teicoplanin, daptomycin, vancomycin, and meropenem, while resistance was high for erythromycin, tetracycline, ampicillin, and ceftriaxone. These findings are similar to the results of a recent Iraqi study which found that microorganisms isolated from DFUs were highly sensitive to imipenem and vancomycin and resistant to doxycycline and ceftriaxone. ${ }^{31}$ The current findings are also similar to the results of Perim et al, whose study in Brazil revealed that imipenem was an effective antibiotic against grampositive and gram-negative bacteria of DFUs. ${ }^{32}$

Previous use of broad-spectrum antibiotics is reported as a common risk factor for the development of highly resistant bacteria. ${ }^{33}$ The higher rate of multidrugresistant bacteria present in the DFUs can be explained by patients experiencing antibiotic therapy prior to surgical debridement. ${ }^{34}$ Antibiotic resistance can also be explained by exposure to multiple courses of antibiotics, which is common among patients with DFUs, because they are usually hospitalized multiple times. ${ }^{35}$ Therefore, the potential presence of such resistant strains puts emphasis on the significance of obtaining optimal specimens from DFUs for culture and sensitivity testing as well as the need to avoid excessive antibiotic therapy which promotes this resistance. $^{36}$

Differences in the results reported by many studies indicate that the patterns of microbial infection are not consistent in patients with diabetic foot infections; therefore, repeated evaluation of microbial characteristics and antibiotic sensitivity is necessary for the selection of appropriate antibiotics. ${ }^{37}$ In the present study, antibiotics such as imipenem, moxifloxacin, teicoplanin, daptomycin, vancomycin, and meropenem caused the highest rate of sensitivity. In terms of the application of imipenem and vancomycin, the current results were in line with those of the study carried out by Perim et al who reported that the prepared cultures were much more sensitive to these antibodies. ${ }^{32}$ Moreover, erythromycin, tetracycline, ampicillin, and ceftriaxone caused the highest rate of resistance to the prepared culture. This finding is in agreement with those reported by Perim et al, who mentioned that erythromycin, tetracycline, and ampicillin antibiotics cause the highest rate of resistance. ${ }^{32}$ In addition, in a similar study, Sivanmaliappan and Sevanan reported that $100 \%$ of Pseudomonas aeruginosa isolates were resistant to ampicillin and norfloxacin, $83.3 \%$ to tetracycline, $66.6 \%$ to gentamicin and imipenem, and $16.6 \%$ to cefotaxime. ${ }^{34}$

In the current study, a highly significant association was observed between the isolated gram-negative microorganisms and higher grades of DFUs $(P<0.001)$. This finding is in agreement with the results of a study by Otta et al in India. ${ }^{38}$ Additionally, the DFU patient's bad knowledge and hygiene practices were significantly associated with gram-negative microorganisms $(P<0.001)$. This finding is in agreement with reports from Kwon and Armstrong's study in South Korea, which documented that bad knowledge and practices of diabetic patients are risk factors for developing foot ulcers with poor prognoses especially if infected with gram-negative bacteria. ${ }^{39}$

In the present study, the mean $\mathrm{Hb}$ of diabetic foot patients was $11 \mathrm{~g} / \mathrm{dL}$, and $48.9 \%$ of diabetic foot patients had anemia. The results of a study by Olgun et al in Turkey revealed that $85.3 \%$ of diabetic foot patients had anemia. ${ }^{40}$ Anemia is common among patients with DFUs and is associated with advanced morbidity of diabetic foot, regardless of its severity. ${ }^{41}$ As seen in HbAlc values, 95.7\% of diabetic foot patients in the present study had poor glycemic control. This finding is similar to the results reported by Chuan et al. ${ }^{42}$

The present study had some limitations. The most remarkable one is related to its small sample size; therefore, generalizing the findings should be done with much caution. The second limitation is related to the fact that cultures for anaerobic bacteria could not be performed. The third limitation is that in studies with a cross-sectional design, it is impossible to infer causality or untangle bidirectional relationships. Future studies are recommended to resolve these limitations.

\section{Conclusion}

DFUs are predominantly caused by gram-positive bacteria, such as $S$. aureus. Moreover, gram-positive bacteria like $S$. aureus were more prevalently isolated from DFUs than gram-negative bacteria like $E$. coli. In addition, imipenem was an effective antibiotic against microorganisms causing DFUs. Various studies on the bacteriology of DFUs have reported results that vary and are often contradictory; therefore, the application of molecular techniques may lead to more accurate microbial characterizations and targeted antibiotic therapy. Thus, it is necessary to evaluate different microorganisms causing DFUs on a routine basis and to know the antibiotic susceptibility patterns of isolates from infected wounds in patients with such lesions. This knowledge is crucial for planning the treatment of these patients with the appropriate antibiotics, reducing resistance patterns, and minimizing healthcare costs. 


\section{Research Highlights}

\section{What Is Already Known?}

DFU is a serious complication associated with diabetes and can have negative effects on quality of life in diabetic patients. According to WHO reports, the incidence of DFU is increasing globally. DFUs are caused by microorganisms; therefore, recognizing the involved microorganisms can be a great step toward developing appropriate antimicrobial therapies.

\section{What This Study Adds?}

DFUs were found to be more prevalent among T2DM males aged above 40 years and among those who had bad knowledge about foot care and hygiene and poor control of HbAlc. S. aureus and E. coli the most prevalent grampositive and gram-negative microorganisms, respectively. The highest level of antibiotic sensitivity was related to imipenem, moxifloxacin, and teicoplanin. Gram-negative microorganisms had highly significant associations with grade of ulcer $(P<0.001)$ and patients' bad knowledge about foot care and hygiene practices $(P<0.001)$. The above-mentioned factors need to be taken into account while developing any antimicrobial therapies.

\section{Authors' Contributions}

ANQ, BMM, TOM contributed to study design, data analysis, and manuscript writing; DMRAA and SOM contributed to critical revisions; all authors read and approved the final manuscript.

\section{Conflict of Interest Disclosures}

The authors declare that they have no conflicts of interest.

\section{Ethical Approval}

Ethical approval was obtained from the Ministry of Higher Education and Scientific Research, Kurdistan Board for Medical Specialties, under the ethical clearance 4573/2010/04/08.

\section{References}

1. Kiadaliri AA, Najafi B, Mirmalek-Sani M. Quality of life in people with diabetes: a systematic review of studies in Iran. J Diabetes Metab Disord. 2013;12(1):54. doi:10.1186/22516581-12-54.

2. Hadadi A, Omdeh Ghiasi H, Hajiabdolbaghi M, Zandekarimi M, Hamidian R. Diabetic foot: infections and outcomes in Iranian admitted patients. Jundishapur J Microbiol. 2014;7(7):e11680. doi:10.5812/jjm.11680.

3. Anvarinejad $M$, Pouladfar $G$, Japoni $A$, et al. Isolation and antibiotic susceptibility of the microorganisms isolated from diabetic foot infections in Nemazee hospital, Southern Iran. J Pathog. 2015;2015:328796. doi:10.1155/2015/328796.

4. Chiwanga FS, Njelekela MA. Diabetic foot: prevalence, knowledge, and foot self-care practices among diabetic patients in Dar es Salaam, Tanzania - a cross-sectional study. J Foot Ankle Res. 2015;8:20. doi:10.1186/s13047-015-0080-y.

5. Al Ayed MY, Ababneh M, Alwin Robert A, et al. Common pathogens and antibiotic sensitivity profiles of infected diabetic foot ulcers in Saudi Arabia. Int J Low Extrem Wounds. 2018;17(3):161-168. doi:10.1177/1534734618793557.

6. Johani K, Fritz BG, Bjarnsholt $\mathrm{T}$, et al. Understanding the microbiome of diabetic foot osteomyelitis: insights from molecular and microscopic approaches. Clin Microbiol Infect. 2019;25(3):332-339. doi:10.1016/j.cmi.2018.04.036.

7. Hatipoglu M, Mutluoglu M, Uzun G, Karabacak E, Turhan V, Lipsky BA. The microbiologic profile of diabetic foot infections in Turkey: a 20-year systematic review: diabetic foot infections in Turkey. Eur J Clin Microbiol Infect Dis. 2014;33(6):871-878. doi:10.1007/s10096-014-2047-5.

8. Mehraj M. A review of Wagner classification and current concepts in management of diabetic foot. Int J Orthop Sci. 2018;4(1):933-935. doi:10.22271/ortho.2018.v4.i1n.133.

9. Mariam TG, Alemayehu A, Tesfaye E, et al. Prevalence of diabetic foot ulcer and associated factors among adult diabetic patients who attend the diabetic follow-up clinic at the University of Gondar Referral Hospital, North West Ethiopia, 2016: institutional-based cross-sectional study. J Diabetes Res. 2017;2017:2879249. doi:10.1155/2017/2879249.

10. Won $\mathrm{SH}$, Chung $\mathrm{CY}$, Park MS, et al. Risk factors associated with amputation-free survival in patient with diabetic foot ulcers. Yonsei Med J. 2014;55(5):1373-1378. doi:10.3349/ ymj.2014.55.5.1373.

11. Deribe B, Woldemichael K, Nemera G. Prevalence and factors influencing diabetic foot ulcer among diabetic patients attending Arbaminch hospital, south Ethiopia. J Diabetes Metab. 2014;5(1):322. doi:10.4172/2155-6156.1000322.

12. Al Benwan K, Al Mulla A, Rotimi VO. A study of the microbiology of diabetic foot infections in a teaching hospital in Kuwait. J Infect Public Health. 2012;5(1):1-8. doi:10.1016/j. jiph.2011.07.004.

13. Abbas ZG, Lutale JK, Archibald LK. The Utility of Gram Stains and Culture in the Management of Limb Ulcers in Persons with Diabetes, Dar es Salaam, Tanzania. Diabetes. 2008;57:A314A314.

14. Ameen SM, Rasul SM. Antimicrobial susceptibility profile of aerobic bacteria collected from diabetic foot ulcer infections, in Sulaimani province. Zanco J Pure Appl Sci. 2018;30(4):115125. doi:10.21271/zjpas.30.4.13.

15. Radji M, Putri CS, Fauziyah S. Antibiotic therapy for diabetic foot infections in a tertiary care hospital in Jakarta, Indonesia. Diabetes Metab Syndr. 2014;8(4):221-224. doi:10.1016/j. dsx.2014.09.006

16. Saber HJ, Daoud AS. Knowledge and practice about the foot care and the prevalence of the neuropathy among a sample of type 2 diabetic patients in Erbil, Iraq. J Family Med Prim Care. 2018;7(5):967-974. doi:10.4103/jfmpc.jfmpc_163_18.

17. Classification and Diagnosis of Diabetes: Standards of Medical Care in Diabetes-2019. Diabetes Care. 2019;42(Suppl 1):S13-s28. doi:10.2337/dc19-S002 .

18. Beyhaghi H, Reeve BB, Rodgers JE, Stearns SC. Psychometric properties of the four-item Morisky green levine medication adherence scale among atherosclerosis risk in communities (ARIC) study participants. Value Health. 2016;19(8):996-1001. doi:10.1016/j.jval.2016.07.001.

19. Muhammad-Lutfi AR, Zaraihah MR, Anuar-Ramdhan IM. Knowledge and practice of diabetic foot care in an inpatient setting at a tertiary medical center. Malays Orthop J. 2014;8(3):22-26. doi:10.5704/moj.1411.005.

20. World Health Organization (WHO). Haemoglobin Concentrations for the Diagnosis of Anaemia and Assessment of Severity. WHO; 2011

21. Jellinger PS, Handelsman $Y$, Rosenblit PD, et al. American association of clinical endocrinologists and American college of endocrinology guidelines for management of dyslipidemia and prevention of cardiovascular disease. Endocr Pract. 
2017;23(Suppl 2):1-87. doi:10.4158/ep171764.appgl.

22. Mendes JJ, Marques-Costa A, Vilela C, et al. Clinical and bacteriological survey of diabetic foot infections in Lisbon. Diabetes Res Clin Pract. 2012;95(1):153-161. doi:10.1016/j. diabres.2011.10.001.

23. Standards of medical care in diabetes--2011. Diabetes Care. 2011;34 Suppl 1(Suppl 1):S11-61. doi:10.2337/dc11-S011.

24. Lipsky BA, Aragón-Sánchez J, Diggle M, et al. IWGDF guidance on the diagnosis and management of foot infections in persons with diabetes. Diabetes Metab Res Rev. 2016;32 Suppl 1:4574. doi:10.1002/dmrr.2699.

25. Sari R, Apridamayanti P, Puspita ID. Sensitivity of Escherichia coli bacteria towards antibiotics in patient with diabetic foot ulcer. Pharmaceutical Sciences and Research (PSR). 2018;5(1):19-24. doi:10.7454/psr.v5i1.3649.

26. Farshad S, Anvarinejad M, Mehrabi Tavana A, et al. Molecular epidemiology of Escherichia coli strains isolated from children with community acquired urinary tract infections. Afr J Microbiol Res. 2011;5(26):4476-4483. doi:10.5897/ AJMR11.285y.

27. Mohammed SI, Mikhael EM, Ahmed FT, Al-Tukmagi HF, Jasim AL. Risk factors for occurrence and recurrence of diabetic foot ulcers among Iraqi diabetic patients. Diabet Foot Ankle. 2016;7:29605. doi:10.3402/dfa.v7.29605.

28. Xie $X, B a o ~ Y, N i ~ L$, et al. Bacterial profile and antibiotic resistance in patients with diabetic foot ulcer in Guangzhou, Southern China: focus on the differences among different Wagner's grades, IDSA/IWGDF grades, and ulcer types. Int J Endocrinol. 2017;2017:8694903. doi:10.1155/2017/8694903.

29. Al-Hemedawi R, Al-Salloum S, Al-Azawi S. Microorganisms isolated from foot ulcers infection of diabetic Iraqi patients. J Fac Med Baghdad. 2006;48(3)297-301.

30. Sekhar S, Vyas N, Unnikrishnan M, Rodrigues G, Mukhopadhyay C. Antimicrobial susceptibility pattern in diabetic foot ulcer: a pilot study. Ann Med Health Sci Res. 2014;4(5):742-745. doi:10.4103/2141-9248.141541.

31. Hasan AY. Antibiotic susceptibility of bacterial isolates from diabetes patients with foot ulcers. Diyala J Pure Sci. 2017;13(3):163-174.

32. Perim MC, Borges Jda C, Celeste SR, et al. Aerobic bacterial profile and antibiotic resistance in patients with diabetic foot infections. Rev Soc Bras Med Trop. 2015;48(5):546-554. doi:10.1590/0037-8682-0146-2015.

33. Harris AD, McGregor JC, Johnson JA, et al. Risk factors for colonization with extended-spectrum beta-lactamaseproducing bacteria and intensive care unit admission. Emerg Infect Dis. 2007;13(8):1144-1149. doi:10.3201/ eid1308.070071.

34. Sivanmaliappan TS, Sevanan M. Antimicrobial susceptibility patterns of Pseudomonas aeruginosa from diabetes patients with foot ulcers. Int J Microbiol. 2011;2011:605195. doi:10.1155/2011/605195.

35. Lipsky BA, Giordano P, Choudhri S, Song J. Treating diabetic foot infections with sequential intravenous to oral moxifloxacin compared with piperacillin-tazobactam/amoxicillinclavulanate. J Antimicrob Chemother. 2007;60(2):370-376. doi:10.1093/jac/dkm130.

36. Lipsky BA, Berendt AR, Cornia PB, et al. 2012 Infectious Diseases Society of America clinical practice guideline for the diagnosis and treatment of diabetic foot infections. Clin Infect Dis. 2012;54(12):e132-173. doi:10.1093/cid/cis346.

37. Singh SK, Gupta K, Tiwari S, et al. Detecting aerobic bacterial diversity in patients with diabetic foot wounds using ERICPCR: a preliminary communication. Int J Low Extrem Wounds. 2009;8(4):203-208. doi:10.1177/1534734609353080.

38. Otta S, Debata N, Swain B. Bacteriological profile of diabetic foot ulcers. CHRISMED J Health Res. 2019;6(1):7-11. doi:10.4103/cjhr.cjhr_117_17.

39. Kwon KT, Armstrong DG. Microbiology and antimicrobial therapy for diabetic foot infections. Infect Chemother. 2018;50(1):11-20. doi:10.3947/ic.2018.50.1.11.

40. Olgun ME, Altuntaş $S$, Sert M, Tetiker T. Anemia in patients with diabetic foot ulcer: effects on diabetic microvascular complications and related conditions. Endocr Metab Immune Disord Drug Targets. 2019;19(7):985-990. doi:10.2174/18715 30319666190111121913.

41. Wright JA, Oddy MJ, Richards T. Presence and characterisation of anaemia in diabetic foot ulceration. Anemia. 2014;2014:104214. doi:10.1155/2014/104214.

42. Chuan F, Zhang M, Yao Y, Tian W, He X, Zhou B. Anemia in patients with diabetic foot ulcer: prevalence, clinical characteristics, and outcome. Int J Low Extrem Wounds. 2016;15(3):220-226. doi:10.1177/1534734616660224. 